medial temporal gyrus, right cuneus, left inferior parietal lobule and left parieto-occipital arcus) at 6 years. The effect of preterm birth in the right junction of paracentral lobule and the precuneus and in the right transverse temporal gyrus shows statistically significant differences between groups $(p=0.001$, positively correlated with thickness at 6 years in the IUGR group and negatively correlated in the non-IUGR group).

Discussion/conclusion Our results indicate that the regional structural reorganization of cerebral cortex after preterm birth differs in IUGR and non-IUGR subjects. Preterm birth affects the higher order association areas with increased thickness or less thinning in IUGR than non-IUGR born children. These cortical changes might underlay the specific functional deficits observed in these children.

\section{IMPROVED DETECTION OF INTRACRANIAL HEMORRHAGE IN TERM AND PRETERM NEONATES USING SUSCEPTIBILITY WEIGHTED IMAGING}

doi:10.1136/archdischild-2012-302724.0196

${ }^{1} \mathrm{~S}$ Sirin, ${ }^{2 B}$ Huening, ${ }^{2} \mathrm{~A}$ Stein, 'SL Goericke, 'A Krasny, ${ }^{2} \mathrm{U}$ Felderhoff-Mueser, 'B Schweiger. 'Institute of Diagnostic and Interventional Radiology and Neuroradiology; ${ }^{2}$ Department of Neonatology, Division of Pediatrics I, University Hospital Essen, Essen, Germany

Background and aims Magnetic resonance imaging (MRI) at term age has been reported to be superior to cranial ultrasound (cUS) in detecting white matter abnormalities. The aim of this retrospective study was to compare sensitivity of MRI using SWI (susceptibility weighted imaging) and cUS in the detection of intracranial hemorrhage.

Methods 68 consecutive term and preterm neonates, who received 3 Tesla MRI of the brain with SWI (Magnetom Skyra, Siemens Healthcare, Erlangen, Germany) around term and serial cUS (Acuson sequoia 512, Siemens Healthcare) during neonatal care, were included in this study between 05/2011 and 02/2012. MRI was performed using a MR-compatible incubator with compatible head coil (LMT nomag, Luebeck, Germany) under sedation. MRI were analyzed by two radiologists independently. Inter-rater agreement was estimated by Cohen's kappa coefficient.

Results MRI and cUS were feasible in all 68 neonates (38 girls, 30 boys, mean gestational age at birth $31.9 \pm 4.5$ weeks (range 23.3-40.7 weeks). MR imaging was done at 40.3 \pm 3.0 weeks (range 30.7-55.7 weeks). Both radiologists independently identified (post-)hemorrhagic alterations in 20 of 68 infants by SWI (inter-rater agreement: $\mathrm{K}=1$ ). In 10 this was in agreement with cUS, but in 4 of them additional intraventricular and/or parenchymal hemorrhagic components were diagnosed by MRI. All patients with suspected intracranial hemorrhage by cUS were confirmed by SWI.

Conclusions We found improved detection of intracranial hemorrhage with high inter-rater agreement by MRI using SWI compared to cUS in term and preterm infants. All hemorrhages diagnosed by cUS could be confirmed by MRI

\section{SERIAL DIFFUSION TENSOR IMAGING DEMONSTRATES: WHITE MATTER MICROSTRUCTURE IN THE PRETERM PERIOD IS NOT RELATED TO GESTATION AT BIRTH}

doi:10.1136/archdischild-2012-302724.0197

1,2BM Huening, 'L Pazderova, 'G Ball, 'N Tusor, 'N Merchant, 'T Arichi, 'JM Allsop, ${ }^{2} \mathrm{U}$ Felderhoff-Müser, ${ }^{1} \mathrm{M}$ Rutherford, ${ }^{1} \mathrm{AD}$ Edwards, 'SJ Counsell. 'Centre for the Developing Brain, Department of Perinatal Imaging, Division of Imaging Sciences \& Bioengineering, King's College London, London, UK; ${ }^{2}$ Division of Pediatrics I, Neonatology, University Children's Hospital, Essen, Germany

Background and aims We have previously shown a dose-dependent effect of prematurity on white matter (wm) microstructure at term equivalent age (TEA). The aim of this study was to determine whether the degree of prematurity at birth is associated with FA values in the early neonatal period.

Methods Inclusion criteria: Preterm birth $<33$ weeks gestational age (GA), serial MRI and DTI, first scan $\leq 33$ weeks post-menstrual age (PMA), second at TEA.

We studied 52 preterm infants, with a median GA at birth of $27+{ }^{1}$ $\left(24^{+3}-32^{+6}\right)$ weeks. DTI data were analysed using tract based spatial statistics (TBSS). Voxel based statistics was performed to assess the correlation between GA at birth and FA, corrected for PMA at scan. Results Scan 1: $31\left(25^{+2}-33\right)$ weeks PMA.

There were no significant correlations between GA at birth and FA in any WM region.

Scan 2: $41^{+1}\left(38^{+6}-44^{+1}\right)$ weeks PMA.

GA at birth was significantly linearly correlated with FA values in the corpus callosum, internal and external capsule, optic radiation, cerebral peduncles, cingulum and inferior longitudinal fasciculus.

Conclusions These data suggest that diffuse wm injury is not an inevitable consequence of preterm birth, and imply there may be a window of opportunity between birth and term eqivalent age where intervention with appropriate treatments may ameliorate the adverse effects of prematurity on wm development.

\section{TRANSPLANTATION FOR PHT IN EARLY CHILDHOOD}

doi:10.1136/archdischild-2012-302724.0198

S Sweet. Pediatric Alllergy, Imunology and Pulmonary Medicine, Washington University, St. Louis, MO, USA

Compared to older children, where Cystic Fibrosis is the most common indication, pulmonary vascular disoders, either related to congenital heart disease or idiopathic pulmonary hypertension, make up $40-50 \%$ of the population of patients undergoing lung transplantation before 6 years of age. Lung transplantation of infants and young children is complicated by challenges in several domains including technical (i.e. airway complications), monitoring (transbronchial biopsies are more difficult to obtain and lung function testing requires sedation), developmental (in particular when oral motor development is delayed) and most importantly level of illness (more than $50 \%$ of infants and young children undergoing lung transplant require mechanical ventilation and/or extracorporeal support prior to transplant. Although improving in recent years, early outcomes lag behind older children and adults (70\% compared to $>80 \%$ ). However 3 and 5 year survival is comparable (65\% and $50 \%$ respectively), perhaps due to a lower incidence of acute and chronic rejection. Many children transplanted in infancy face developmental delays, most likely due to pretransplant insults. Nonetheless, excellent long term outcome is possible and should become increasingly likely with improvements in pretransplant support and management of posttransplant complicaitons.

\section{A HYBRID GENOME-KINOME HIGH-THROUGHPUT SCREEN REVEALS NOVEL MITOTIC TUMOR SUPPRESSOR SIGNALING AXIS}

doi:10.1136/archdischild-2012-302724.0199

'G Nalepa, ${ }^{2} \mathrm{~J}$ Barnholtz-Sloan, 'AS Lehmann, 'FC Yang, 'J Renbarger, 'DW Clapp. ${ }^{1}$ Indiana University School of Medicine, Indianapolis, IN; ${ }^{2}$ Case Western Reserve University Comprehensive Cancer Center, Cleveland, $\mathrm{OH}$, USA

Faithful cell division maintains genomic stability and prevents cancer. Our cells employ well-orchestrated signaling cascades to ensure meticulous segregation of the genome during mitosis. Failure of these checkpoint mechanisms jeopardizes genome integrity and promotes evolution of cancer cells. 
Here, we took a systems biology approach to characterize phosphatases regulating mitosis. We performed a genome-wide RNAi screen targeting all human phosphatases.

We discovered several novel mitotic phosphatases, including CDKN3, and we have shown that CDKN3 inactivates cyclindependent kinases at the exit from mitosis by dephosphorylating Thr-161 of CDC2. We demonstrated that CDKN3 and CDC2 colocalize on centrosomes during mitosis and that loss of CDKN3 disrupts centrosome maintenance. We analyzed a phosphoproteome landscape of

CDKN3-deficient cells to reveal that CDKN3 knockdown leads to abnormal phosphorylation of multiple downstream cell cycle proteins, including CK $\beta$. We have shown that CK $\beta$ phosphorylated at Ser-209 regulates the spindle checkpoint and localizes to centrosomes during mitosis. We confirmed that CDKN3 is required for mitosis in primary human brain stem cells, and we found that CDKN3 is lost in the glioblastoma multiforme brain tumors.

In summary, we have discovered a novel CDKN3/CDC2/CK $\beta$ tumor suppressor signaling axis. Our findings have diagnostic and therapeutic importance in cancer. Our discoveries enhance our comprehension of the cross-talk between mitotic phosphorylation cascades that maintain genomic stability. This signaling axis is a viable anti-cancer target in glioblastoma multiforme and other malignancies. Pre-clinical and clinical trials of small molecules targeting this signaling axis may lead to discoveries of novel anti-cancer chemotherapy strategies.

\section{CIRCULATING PRO-ENDOTHELIN-1 (CT-PROET-1) AND PULMONARY MORBIDITY IN NEWBORN INFANTS}

doi:10.1136/archdischild-2012-302724.0200

1,2S Wellmann, ${ }^{2} \mathrm{~J}$ Benzing, ${ }^{1} 0$ Stabile, ${ }^{2} \mathrm{G}$ Szinnai, ${ }^{3} \mathrm{NG}$ Morgenthaler, ${ }^{2} \mathrm{SM}$ Schulzke, ${ }^{3} \mathrm{C}$ Bührer. 'University Hospital Zurich, Zurich; 'University Children's Hospital Basel, Basel, Switzerland; ${ }^{3}$ Charité University Medical Centre, Berlin, Germany

Background and aims Plasma concentrations of endothelin-1 (ET-1), a potent pulmonary vasoconstrictor with a short serum half-life, can be estimated by measuring its stable by-product, C-terminal pro-Endothelin-1 (CT-proET-1). To investigate the association between CT-proET-1, gestational age, and pulmonary morbidity in newborn infants.

Methods A prospective cross-sectional study at two tertiary university hospitals was conducted. CT-proET-1 concentrations (pmol/L) were determined in plasma samples of 293 newborn infants (gestational age 24-41 weeks) at birth and on day 3 of life with BRAHMS KRYPTOR automated immunoflorescent assay.

Results At birth, CT-proET-1 concentrations were unrelated to birth weight and gestational age. Venous umbilical cord CT-proET-1 concentrations were consistently higher than matched arterial ones (M 148 vs. $134 \mathrm{pmol} / \mathrm{L}, \mathrm{p}<0.001$ ), but both values were closely related $\left(R_{s}=0.745, p<0.001\right)$. There was large postnatal increase of CT-proET-1 in infants with pulmonary morbidity. Days of mechanical ventilation, continuous positive airway pressure (CPAP), and oxygen supplementation correlated each with CT-proET-1 level on day $3\left(R_{s} 0.566,0.658\right.$, and 0.819 , respectively, for all $\left.p<0.001\right)$.

Conclusions In term and preterm newborn infants, pulmonary compromise is associated with increased concentrations of circulating CT-proET-1.

\section{EFFICACY OF MONOTHERAPY WITH INHALED NITRIC OXIDE VERSUS COMBINATION WITH ORAL SILDENAFIL IN PERSISTENT PULMONARY HYPERTENSION OF THE NEWBORN}

SS Oguz, HG Kanmaz, E Dizdar Alyamac, FN Sari, 0 Erdeve, U Dilmen. Zekai Tahir Burak Maternity Teaching Hospital Neonatology Unit, Ankara, Turkey

Objective To evaluate the efficacy of combined therapy, sildenafil and inhaled nitric oxide versus monotherapy with inhaled nitric oxide in Pulmonary Hypertension of the Newborn.

Study Design A retrospective study.

Patients and Methods Newborn infants (gestational age greater than 34 weeks) who were presented with pulmonary hypertension from December 2008 to December 2010 were evaluated. Group I $(n=14)$ received monotherapy with iNO and Group II $(n=9)$ received combination therapy with iNO and oral sildenafil. Main outcome was to compare the duration of iNO therapy between groups.

Results Demographic characteristics were similar between the groups. As compared with the groups, combined therapy group had higher mean (SD) age of NICU admission (5.1 $\pm 8.2 \mathrm{~h}$ vs. $21.3 \pm 36, \mathrm{~h}$, $\mathrm{p}=0.01)$. Combination therapy was associated with early weaning of iNO (4.8 \pm 1.5 vs. $13.5 \pm 7.6$ hours). The result showed that there is a reduced need for iNO therapy compared to monotherapy $(75 \pm 44.6$ vs. $112 \pm 95.2)$, however, the difference was insignificant $(p=0.36)$. The incidence of mortality and outcomes were statistically insignificant between the groups ( $p>0.05)$.

Conclusions Combination therapy is significantly more effective in weaning of iNO and reduces the need for iNO therapy that is relatively expensive.

\section{SURFACTANT AND INHALED NITRIC OXIDE FOR SEVERE RESPIRATORY DISTRESS COMPLICATED WITH PERSISTENT PULMONARY HYPERTENSION AMONG INFANTS BELOW 32 WEEKS GA}

doi:10.1136/archdischild-2012-302724.0202

'A Adamczak, ${ }^{2} \mathrm{~S}$ Gajos, ${ }^{2} \mathrm{~A}$ Mejsak, ${ }^{3} \mathrm{~T} A$ Merritt, 'J Gadzinowski, 'J Mazela. ${ }^{1}$ Neonatology; ${ }^{2}$ Neonatal Research Club, Poznan University of Medical Sciences, Poznan, Poland; '3Pediatrics, Loma Linda Medical Center, Loma Linda, CA, USA

Introduction RDS can be complicated by PPHN due to intrauterine or postnatal prolonged hypoxia regardless of surfactant replacement therapy (SRT). Use of iNO therapy is not approved for premature infants $<34$ weeks, nevertheless in most severe cases of PPHN use of iNO has been reported.

Aim The aim of this study was to analyze outcomes of infants with severe RDS treated with SRT and iNO in comparison to those treated with SRT only.

Methods Medical records from January 2008 till December 2010 from a level III NICU were analyzed. Only infants $<32$ weeks ga and treated with SRT for RDS were included in the study divided in two groups: treated with SRT+iNO (PPHN based on $\mathrm{SaO}_{2}$ differences and echocardiography) and SRT only. Data were analyzed according to maternal history: intrauterine infection, PROM, and clinical outcomes: pneumonia, NEC, ROP, BPD, IVH.

Results 309 premature infants $<32$ weeks gestation were treated with SRT, with 54(17\%) treated with iNO due to PPHN. There were significant higher ratios of intrauterine infection and early pneumonia in SRT+iNO in 2008 and 2009. The neonatal outcomes showed consistent higher incidence of ROP and PVL for the SRT+iNO group in these same years. The BPD rate remained unchanged at $20 \%$ and mortality ranged from 18 to $48 \%$.

Conclusions Severe PPHN was likely caused by ineffective SRT due to presence of inflammation and possible surfactant inactivation. Inhaled NO improved oxygenation and decreased signs of PPHN but did not influence rate of BPD.

\section{THE AVON LONGITUDINAL STUDY OF PAREANTS AND CHILDREN (ALSPAC) - WHAT DID WE LEARN?}

doi:10.1136/archdischild-2012-302724.0203 